\title{
COMBINATION OF PEUTZ-JEGHERS SYNDROME AND PLATELET STORAGE POOL DEFICIENCY: A CASE REPORT
}

\author{
Radislav Nakov ${ }^{1}$, Ventsislav Nakov ${ }^{1}$, Borislav Vladimirov ${ }^{2}$, Antoaneta Zarkova ${ }^{3}$ \\ ${ }^{1}$ Medical University, Sofia, Bulgaria \\ 2 Supervisor; Gastroenterology Clinic, University Hospital Tsaritsa Yoanna-Isul, Sofia, Bulgaria \\ ${ }^{3}$ Supervisor; National Specialized Hospital for Active Treatment of Hematological Diseases, Sofia, Bulgaria
}

\section{Abstract}

Conclusion: This case illustrates the importance of taking into account the possibility for coexistence of Peutz-Jeghers syndrome (PJS) and platelet storage pool deficiency (PSPD) because this combination can lead to serious risks for the patient following invasive procedures.

Introduction: PJS is an autosomal dominant inherited disorder characterized by intestinal hamartomatous polyps in association with a distinct pattern of skin and mucosal macular melanin deposition. PSPD is a rare platelet abnormality that causes a mild to moderate bleeding disorder.

The Aim: We observed a patient with both PJS and PSPD.

The Case: A 30-year old female was presented with complaints of colicky pain in the abdomen, easy bruising and abnormally heavy and prolonged menstruation. The endoscopic examinations found multiple polyps in the stomach, small intestine and colon. The combination of histopathologically verified hamartomatous polyps and patient's mucocutaneous melanotic pigmentation suggested the diagnosis of Peutz-Jeghers syndrome. During the polypectomy we noticed an excessive bleeding. The laboratory tests showed that platelet aggregation after stimulation with ADP and epinephrine was reduced. Moreover, only partial agglutination after stimulation with ristocetin was observed. These results suggested a deficiency of platelet granules and allowed us to diagnose PSPD in our patient.

Key words: Peutz-Jeghers syndrome, platelet storage pool deficiency.

\section{Introduction}

Peutz-Jeghers syndrome (PJS) is an autosomal dominant inherited disorder characterized by intestinal hamartomatous polyps in association with a distinct pattern of skin and mucosal macular melanin deposition [1]. We observed a patient with both PJS and platelet storage pool deficiency (PSPD) - a rare platelet abnormality that causes a mild to moderate bleeding disorder. The risk of bleeding after polypectomy and the possible complications pose a therapeutic challenge to the clinician.

\section{Case report}

A 30-year old female was admitted for the first time to the hospital in 2003 with complaints of colicky pain in the abdomen, bloating and abdominal discomfort. She had also a history of easy bruising and abnormally heavy and prolonged menstruation. She had never had any surgical procedures including dental extraction. The patient had no other significant past and family history. Moreover, she denied alcohol use, herbal or overthe-counter medications and allergies. The physical examination revealed bruising on the thighs and hyperpigmentation of the labial, buccal and genital mucosa (Figure 1).

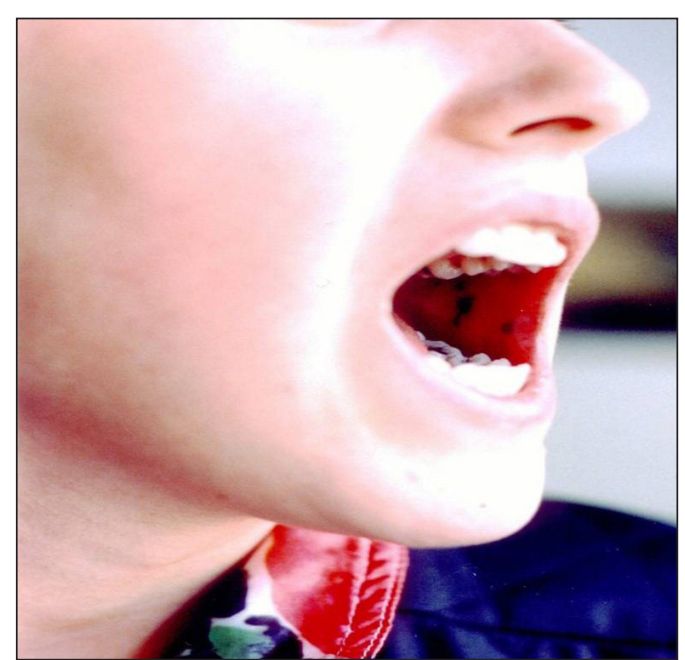

Figure 1. Hyperpigmentation of the buccal mucosa. 
The laboratory tests and the abdominal ultrasonography did not show anything significant. The endoscopic examinations (fibrogastroscopy, colonoscopy and single balloon enteroscopy) found multiple polyps in the stomach, small intestine and colon (Figure 2). The biggest ones were removed. During the polypectomy we noticed an excessive bleeding and because of that we used argon plasma coagulation (APC) and terlipressin to control it.

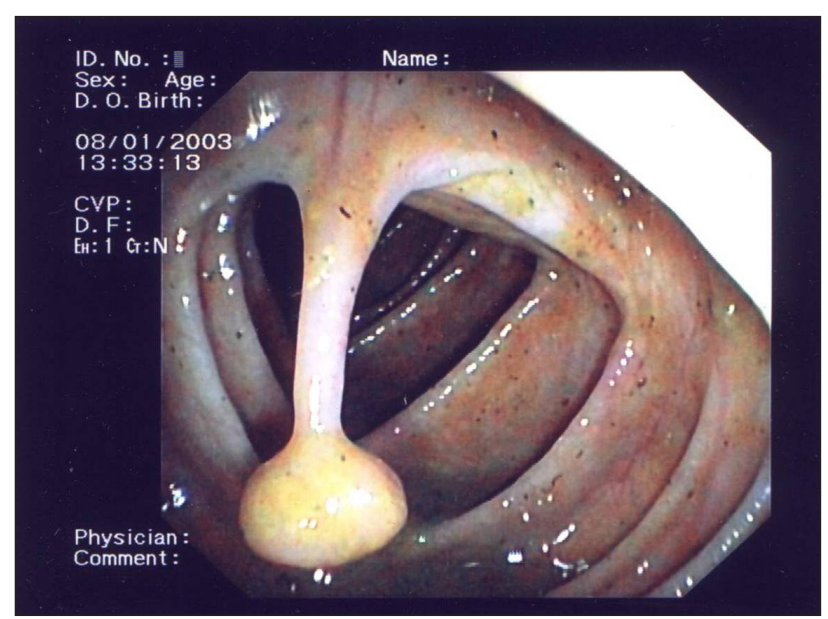

Figure 2. Endoscopic examination of the intestines.

Multiple sections from the polyps that were examined showed features of hamartomatous polyps composed of glands separated by a band of smooth muscles. There was no evidence of atypia or malignancy. The combination of histopathologically verified hamartomatous polyps and mucocutaneous melanotic pigmentation suggested the diagnosis of Peutz-Jeghers syndrome. We could not carry out a mutation analysis for STK 11/LKB 1 gene due to lack of resources.

In the following years (2003-2008) the patient was hospitalized anually for removal of polyps from the gastrointestinal tract (GIT). During one of these polypectomies in 2008, she had a massive haemorrhage from one of the polypectomy sites in the jejunum which required additional endoscopic haemostasis, application of terlipressin, and hemotransfusions. In view of this situation, we consulted a specialist in hematology and performed additional laboratory tests. The latter found prolonged bleeding time (13s). The other parameters of the complete blood count and the coagulation screen (prothrombin time, activated partial thromboplastin time, Clauss fibrinogen and thrombin time) were normal.

Both ADP and epinephrine-induced aggregation showed presence of primary, and absence of secondary aggregation wave. Moreover, only partial agglutination after stimulation with ristocetin was observed. These results suggested a deficiency of platelet granules and al- lowed us to diagnose platelet storage pool deficiency in our patient.

In the period 2009-2013 the patient was followed-up and polypectomies were performed every year following preventive application of fresh frozen plasma and platelets.

\section{Discussion}

Peutz-Jeghers syndrome is a rare autosomal dominant disorder with variable penetrance. Its frequency is between 1 case per 60000 people and 1 case per 300 000 people [2]. Patients with PJS have a 15 -fold increased risk of developing intestinal cancer compared with the general population [3-5]. In addition, extraintestinal malignancies have also been associated with PJS, including adenoma malignum of the cervix, Sertoli cell tumors, and sex cord tumors with annular tubules [6].

Platelet storage pool deficiencies (PSPDs) are rare inherited bleeding disorders affecting both males and females, occurring in families as well as sporadically. These abnormalities are characterized by a reduction in the number and content of certain types of platelet granules called dense granules and alpha granules. The dense granules in platelets serve as a storage pool for adenosine triphosphate (ATP), adenosine diphosphate (ADP), serotonin, calcium, and pyrophosphate, which are secreted when platelets are activated [7,8]. Alpha-granules contain numerous proteins involved in platelet adhesion (von Willebrand factor (VWF), P-selectin, fibronectin, fibrinogen), coagulation factors (factors $\mathrm{V}$ and XIII), growth factors (platelet-derived growth factor, transforming growth factor- $\beta$ ), and platelet factor- 4 . The inheritance of an isolated platelet storage pool deficiency is thought to be autosomal dominant, but the penetrance of the gene vary from person to person [9].

PSPDs can also form part of other inherited conditions including Hermansky-Pudlak syndrome, Chediak-Higashi syndrome, which are autosomal recessive conditions, Wiskott-Aldrich syndrome, an X-linked recessive condition, and thrombocytopenia-absent radius (TAR) syndrome [10].

In the available literature we did not find any evidence of combination of PJS and PSPD. In our case, serious therapeutic problems arose, because the growing hamartomatous polyps in PJS had to be removed endoscopically. The serious risk of bleeding due to the presence of PSPD could lead to complications and even to situations endangering patient's life. In our patient, the administration of fresh frozen plasma (FFP) and platelets largely prevented a severe haemorrhage. However, the bleeding we observed after a snear polypectomy was more excessive than normal. This required APC and ad- 
ministration of terlipressin.

In conclusion, this case illustrates the importance of taking into account a possibility for such a combination (PJS and PSPD), because it can lead to serious risks for the patient following invasive procedures.

\section{References}

1. Calva D, Howe JR. Hamartomatous polyposis syndromes. Surg Clin North Am. 2008; 88(4): 779-817.

2. Brosens LA, van Hattem WA, Jansen M, de Leng WW, Giardiello FM, Offerhaus GJ. Gastrointestinal polyposis syndromes. Curr Mol Med. 2007; 7(1): 29-46.

3. Giardiello FM, Welsh SB, Hamilton SR, et al. Increased risk of cancer in the Peutz-Jeghers syndrome. N Engl J Med. 1987; 316 (24): 1511-4.

4. Boardman LA, Thibodeau SN, Schaid DJ, et al. Increased risk for cancer in patients with the Peutz-Jeghers syndrome. Ann Intern Med. 1998; 128(11): 896-9.

5. Gruber SB, Entius MM, Petersen GM, et al. Pathogenesis of adenocarcinoma in Peutz-Jeghers syndrome. Cancer Res. 1998; 58(23): 5267-70.

6. Boardman LA, Pittelkow MR, Couch FJ, et al. Association of Peutz-Jeghers-like mucocutaneous pigmentation with breast and gynecologic carcinomas in women. Medicine (Baltimore). 2000; 79(5): 293-8.

7. Online Mendelian Inheritance in Man, $\mathrm{OMIM}^{\circ}$. Johns Hopkins University, Baltimore, MD. MIM Number: 185050: 03/03/2009. http://omim.org/.

8. Sandrock K, Zieger B. Current strategies in diagnosis of inherited storage pool defects. Transfus Med Hemother. 2010; 37(5): 248-258.

9. Rahman S, Myers J, Gilham J, Fitzmaurice R, Johnston T. Post partum haemorrhage secondary to uterine atony, complicated by platelet storage pool disease and partial placenta diffusa: a case report. Cases J [Internet]. 2008 [cited 2013 Oct 15]; 1: 393. http://www.ncbi.nlm.nih.gov/ pmc/articles/PMC2614952/pdf/1757-1626-1-393.pdf.

10. Riley RS. Platelet storage pool diseases. Virginia Commonwealth University. http://www.pathology.vcu.edu/ clinical/coag/SPD.pdf. [cited 2013 Oct 15]. 